\title{
Conformations of confined biopolymers
}

\author{
Frederik Wagner, ${ }^{1}$ Gianluca Lattanzi, ${ }^{2}$ and Erwin Frey ${ }^{1}$ \\ ${ }^{1}$ Arnold Sommerfeld Center for Theoretical Physics (ASC) and Center for NanoScience (CeNS), \\ Ludwig-Maximilians-Universität München, Theresienstraße 37, D-80333 München, Germany \\ ${ }^{2}$ Department of Medical Biochemistry, Biology and Physics, TIRES-Center and INFN, Università di Bari, \\ Piazza Giulio Cesare 11, 70124 Bari, Italy
}

(Received 19 July 2006; revised manuscript received 26 March 2007; published 31 May 2007)

\begin{abstract}
Nanoscale and microscale confinement of biopolymers naturally occurs in cells and has been recently achieved in artificial structures designed for nanotechnological applications. Here, we present an extensive theoretical investigation of the conformations and shape of a biopolymer with varying stiffness confined to a narrow channel. Combining scaling arguments, analytical calculations, and Monte Carlo simulations, we identify various scaling regimes where master curves quantify the functional dependence of the polymer conformations on the chain stiffness and strength of confinement.
\end{abstract}

DOI: 10.1103/PhysRevE.75.050902

What is the effect of confinement on the shape of a biopolymer? With recent advances in visualizing and manipulating macromolecules on ever shrinking length scales, an answer to this question has gained increasing importance. In the crowded environment of a cell the conformations of cytoskeletal filaments are highly constrained by other neighboring macromolecules. This confinement largely alters the viscoelastic response of entangled biopolymer solutions $[1,2]$. There is growing interest in manufacturing nanostructures such as nanopores [3] and nanochannels [4,5] for investigating and manipulating DNA with improved technologies aiming toward smaller and smaller structures. Hence an improved understanding of the effect of confinement on biopolymer conformations has potential implications for the design of nanoscale devices in biotechnological applications. Similarly, microfluidic devices have been used to explore confinement effects on actin filaments and DNA [6,7]. What makes the confinement of biopolymers both a challenging and interesting problem is that biopolymers, unlike their synthetic counterparts, are generally stiff on a length scale much larger than their monomer size. The persistence length $\ell_{p}$, the scale below which bending energy dominates over thermal fluctuations, is approximately $50 \mathrm{~nm}$ for DNA [8] and $16 \mu \mathrm{m}$ for F-actin [9]. Depending on whether the contour length $L$ is smaller or larger than the persistence length we may distinguish between stiff and flexible chains.

For cellular systems as well as for nanoscale devices, biopolymers are confined on length scales comparable with their persistence length $\ell_{p}$ such that the polymer's intrinsic bending stiffness plays a decisive role for its conformations. For simplicity, consider a cylindrical tube of diameter $d$. Upon balancing the bending stiffness of a chain with thermal energy, Odijk [10] has identified a length $L_{d}$ measuring the typical distance between successive deflections of the chain, $L_{d}^{3} \sim d^{2} \ell_{p}$; see Fig. 1. This suggests to use the number of collisions $c=L / L_{d}$ per filament length $L$ as a natural dimensionless parameter to measure the strength of confinement and $\varepsilon=L / \ell_{p}$ to measure the flexibility of a polymer. The physics in the strong confinement regime $(c \gg 1)$ is genuinely different from the regime where the radius of gyration $R_{\mathrm{G}}$ of a long flexible chain (with $L \gg \ell_{p}$ ) becomes compa- rable to $d$. In the latter case of weak confinement of a flexible chain the shape of the polymer is distorted due to selfavoidance between distant segments along the polymer chain. Then, according to de Gennes' blob picture [11], we may represent the conformation of the polymer as a linear chain of nonpenetrating spheres of radius $d$, where each sphere is described by Flory's theory. This picture results in the following scaling law for an extension of the polymer along the tube axis:

$$
R_{\|} \sim L\left(\ell_{p} / d\right)^{2 / 3} .
$$

For strong confinement the Odijk length becomes the analog of the blob size, below which the polymer may be considered as free. Thus the fraction of contour length stored in thermal undulations decreases with decreasing tube dimensions [10]:

$$
\left(L-R_{\|}\right) / L \sim L_{d} / \ell_{p} .
$$

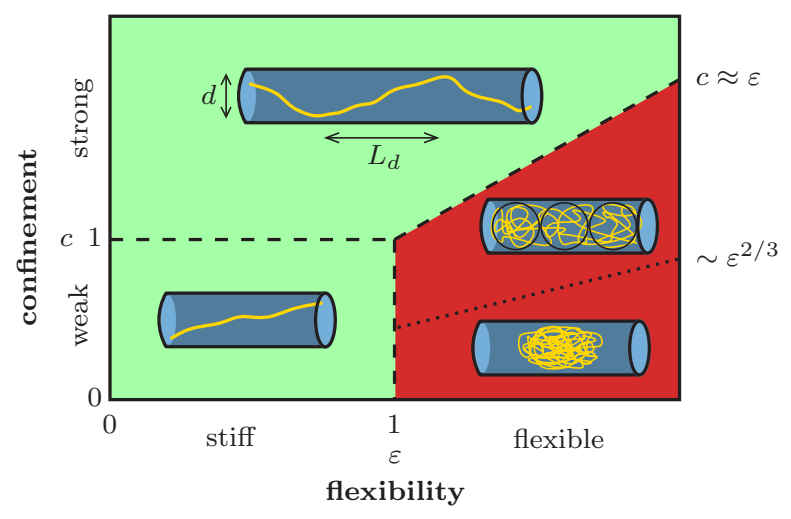

FIG. 1. (Color online) Scaling regimes for confined biopolymer conformations as a function of polymer flexibility $\varepsilon=L / \ell_{p}$ and confinement strength $c=L / L_{d}$. In the flexible regime $(\varepsilon \gg 1)$ two scaling regimes are known [dark gray (red)]: free coil behavior for weak confinement and de Gennes scaling for intermediate confinement. In the parameter range most relevant for biopolymers [light gray (green)], one has to distinguish between weak confinement of stiff polymers and strong confinement for chains of arbitrary stiffness. 
This scaling law should apply equally well for stiff and flexible chains as long as the collision parameter $c$ is sufficiently large. For flexible chains, $\varepsilon \gg 1$, this is the case if the deflection length $L_{d}$ is less than the persistence length $\ell_{p}$. For stiff chains, $\varepsilon \ll 1$, the deflection length has to become smaller than the total filament length before there is any stretching. There is an additional regime of weak confinement $(d \leq L$ $\leq L_{d}$ ) where the average orientation of the filament becomes aligned with the tube axis. These various scaling scenarios are summarized in Fig. 1.

The purpose of this Rapid Communication is to go beyond this qualitative scaling picture and provide a quantitative study of the conformations of biopolymers in confined geometry. Our focus is on the parameter range that is most relevant for cellular systems and nanoscale devices [light gray (green) region in Fig. 1], where self-avoidance effects may safely be neglected. For specificity, we consider a wormlike chain in a soft harmonic potential of cylindrical symmetry and strength $\gamma$. Indeed, the harmonic potential is the simplest model to represent a tubelike confinement and it has the advantage of being amenable to analytic calculations. Thus the Hamiltonian for the contour $\mathbf{r}(s)$ parametrized in terms of the arclength $s$ reads

$$
\mathcal{H}[\mathbf{r}(s)]=\frac{\kappa}{2} \int_{0}^{L} d s\left(\frac{\partial^{2} \mathbf{r}(s)}{\partial s^{2}}\right)^{2}+\frac{\gamma}{2} \int_{0}^{L} d s \mathbf{r}_{\perp}^{2}(s),
$$

where $\kappa=\ell_{p} k_{\mathrm{B}} T$ is the bending stiffness and $\mathbf{r}_{\perp}=(x, y)$ are the components of the contour perpendicular to the tube axis.

A variety of analytical results have been obtained so far for unconfined wormlike chains. For instance, the tangenttangent correlation functions [12] and moments of the endto-end distributions have been calculated exactly $[13,14]$. Further results like the probability distribution function of the end-to-end distance $\mathbf{R}$ have been calculated for stiff chains [15] within the weakly bending rod (WBR) approximation, where one considers only small transverse bending fluctuations with respect to a straight countour $\mathbf{r}(s)$ $\approx\left(\mathbf{r}_{\perp}(s), s\right)$. For confined chains the WBR limit amounts to assume that the filament is aligned with the tube axis, and may be employed to study the asymptotic regime of strong confinement, $L_{d} \ll L$. Then, using the equipartition theorem one obtains for the correlation function of the transverse undulations in Fourier space:

$$
\langle x(k) x(-k)\rangle=\frac{k_{\mathrm{B}} T}{\kappa\left(k^{4}+4 L_{d}^{-4}\right)},
$$

where $L_{d}=(4 \kappa / \gamma)^{1 / 4}$. This simple result forms the basis for all subsequent analytical calculations. For example, it implies that the local transverse mean-square displacement is given by $\left\langle\mathbf{r}_{\perp}^{2}\right\rangle=L_{d}^{3} / 4 \ell_{p}$.

In general, however, analytical calculations are not feasible. In order to investigate the full range of parameters we employed a standard Monte Carlo (MC) scheme using a discretized version of the wormlike chain model. This allows us to go beyond the WBR limit and calculate a range of observables which are directly accessible in single-molecule experiments. The polymer was represented by a chain of $N$ seg-

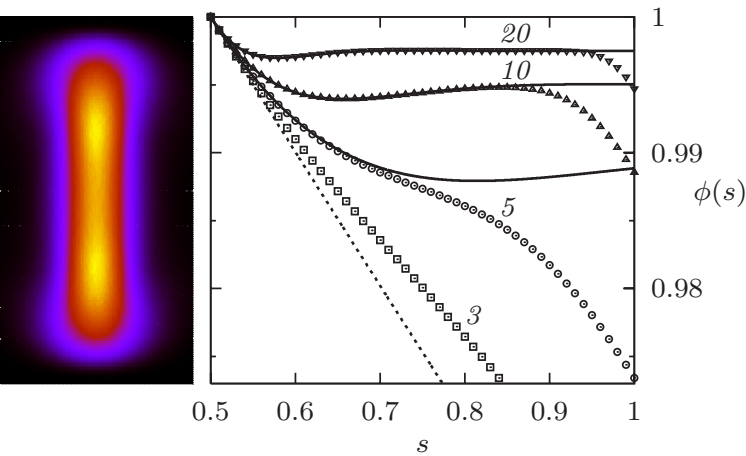

FIG. 2. (Color online) Left: marginal probability distribution function of polymer configurations in a plane containing the tube axis for $c=4$ and $\varepsilon=2$. The probability density increases from dark to light colors. Right: normalized tangent-tangent correlation function $\phi(s)=\langle\mathbf{t}(s) \cdot \mathbf{t}(0.5)\rangle$ for $\varepsilon=0.1$ and various confinements ranging from $c=3$ to $c=20$. MC simulations are represented by symbols, whereas the solid lines show the analytical approximations of $\phi(s)$ as from Eq. (5). Dashed line: exponential decay of an unconfined chain.

ments $\mathbf{t}_{i}$ approximating the continuous contour. The inextensibility constraint was imposed along the whole polymer by fixing the segments length to the value $L / N$. The cylindrical symmetric harmonic potential was calculated at the end points of each segment.

During the simulation, both ends of the polymer were assumed to be completely free, in both position and orientation. The initial configuration was chosen sufficiently close to full stretching, in order to ensure a fast convergence of the $\mathrm{MC}$ algorithm. A new configuration was generated by changing the orientation of a randomly chosen segment and accepted according to the standard Metropolis algorithm. We have not considered effects resulting from self-avoidance, which is not important for strong confinement and in the stiff limit, and is negligible even in the flexible limit if the number of segments is below $N \approx 500$. At least $10^{6} \mathrm{MC}$ steps per segment were performed, to obtain averages and statistical errors. Our MC procedure was validated by evaluating known quantities for polymers in bulk [16].

In a typical experimental setup measuring the shape of a biopolymer in confined geometry, the filaments are labeled with some fluorescent dye and recorded over the time resolution window of the camera. This results in an intensity profile for the emitted light that corresponds, in our theoretical model, to the marginal probability distribution function of the positions of the constituent segments in a plane containing the tube axis. Figure 2 shows this function as obtained from our MC simulations for intermediate values of the collision and stiffness parameters: $c=4$ and $\varepsilon=2$. This picture nicely illustrates the shape of a biopolymer in confined geometry. Given sufficient experimental resolution it may even be possible to resolve the bimodality of the distribution clearly visible in the MC data.

Beyond such a qualitative impression of the shape of the polymer, other more quantitative measures may characterize better its conformations. We start our discussion with the tangent-tangent correlation function $\phi(s)=\langle\mathbf{t}(s) \cdot \mathbf{t}(0.5)\rangle$, measured from the center of the filament. Figure 2 shows the 


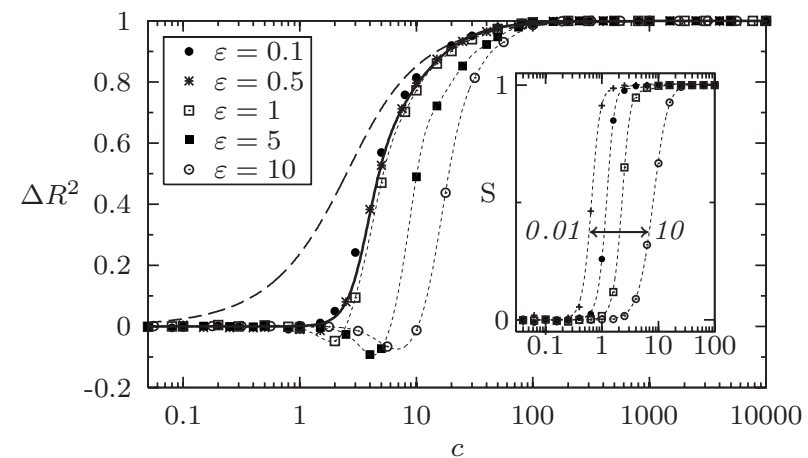

FIG. 3. Reduced mean-square end-to-end distance $\Delta R^{2}$ as a function of the collision parameter $c$ for a series of flexibilities $\varepsilon$ indicated in the graph. The universal scaling curve (solid line) asymptotes the analytical result (long-dashed line) in the limit of strong confinement. Short-dashed lines are guides to the eye for $\varepsilon$ $>1$. Inset: orientational order parameter $S$ as a function of $c$ for $\varepsilon$ $=0.01,0.1,1,10$.

results of our MC simulations in the stiff regime for $\varepsilon=0.1$ and a range of collision parameters $c$. For small $c$ the data show the expected exponential decay of a free filament. For $c \geq 1$ confinement effects become visible and deflections start to affect the correlations for distances comparable to the Odijk length scale $1 / c$. For strong confinement, $\phi(s)$, after an initial exponential decay and a shallow minimum at $s-0.5 \approx 1 / c$, reaches a broad plateau before correlations decay again in a small boundary layer of size $1 / c$. All these features, but the boundary layer effect, are well captured by a formula (solid line in Fig. 2) easily obtained in the WBR approximation from Eq. (4):

$$
\begin{aligned}
\left\langle\mathbf{t}(s) \cdot \mathbf{t}\left(s^{\prime}\right)\right\rangle= & 1-\frac{\varepsilon}{2 c}\left[\sqrt{2} \exp \left(-\frac{\left|s-s^{\prime}\right|}{L} c\right)\right. \\
& \left.\times \sin \left(\frac{\left|s-s^{\prime}\right|}{L} c-\frac{\pi}{4}\right)+1\right] .
\end{aligned}
$$

The quantity which best characterizes the elongation of the polymer is the mean-square end-to-end distance $\left\langle R^{2}\right\rangle$. As already noted, this is exactly known for unconfined chains, $\left\langle R^{2}\right\rangle_{0}=2 L^{2} \varepsilon^{-2}\left(\varepsilon-1+e^{-\varepsilon}\right)$, but can be calculated for strong confinement in the WBR approximation. We find

$$
\frac{\left\langle R^{2}\right\rangle_{c}}{L^{2}}=1-\frac{\varepsilon}{2 c}\left\{1+\frac{1}{c^{2}}\left[1-\sqrt{2} e^{-c} \sin \left(c+\frac{\pi}{4}\right)\right]\right\} \text {. }
$$

The validity of this formula, obtained in the WBR limit, extends to the case of weak confinement: this result correctly recovers the exact result for unconfined chains in the stiff limit. Upon defining the reduced end-to-end distance

$$
\Delta R^{2}(c)=\left(\left\langle R^{2}\right\rangle_{c}-\left\langle R^{2}\right\rangle_{0}\right) /\left(L^{2}-\left\langle R^{2}\right\rangle_{0}\right),
$$

one finds that this quantity is a function of the collision parameter $c$ only. This suggests to look for a data collapse in the MC data. In fact, as can be inferred from Fig. 3, the reduced end-to-end distance is a function of the collision parameter only once the flexibility parameter falls below $\varepsilon$ $\approx 1$-i.e., in the stiff regime. This implies that there is a single master curve characterizing the shape of a stiff polymer.

The analytical results capture the MC results only in the limit of very strong confinement. This is due to the fact that the WBR approximation assumes the filament to be perfectly aligned with the tube axis, which is strictly valid only if $c \gg 1$. In the regime of weak confinement the primary effect of the geometric constraints is to align the filament with the tube axis. We expect this alignment to start once the filament length (more precisely the end-to-end distance) becomes comparable with the tube diameter-i.e., for $c^{3} \sim \varepsilon$. To render this statement quantitative we define the orientational order parameter

$$
S=\frac{1}{2}\left(3\left\langle\cos ^{2} \vartheta\right\rangle-1\right)
$$

where $\vartheta$ is the angle of $\mathbf{R}$ with respect to the tube axis. In fact, as can be inferred from the inset of Fig. 3, there is an intermediate confinement regime where the onset of orientational order precedes filament elongation. This has important implications for the confinement of biopolymers like F-actin and microtubules in artificial channels and cellular systems. For instance, one estimates that for F-actin with $L=2 \mu \mathrm{m}$ this window in tube dimensions ranges from $d=2 \mu \mathrm{m}$ down to $d \approx 0.4 \mu \mathrm{m}$. In this window the free energy cost for confinement will not be given by the Odijk estimate $F \sim k_{B} T c$ but by the constraint on the orientational degrees of freedom-i.e., $F \sim k_{B} T \ln (L / d)$. This intermediate regime becomes less pronounced with increasing flexibility. Actually, even very long (self-avoiding) polymers are known to have an instantaneous prolate shape $[17,18]$. This anisotropy in the radius of gyration tensor is rather due to entropy effects [19] than the energy of bending as for stiff biopolymers discussed here. Despite the different physical origin its consequences are that also flexible polymers in confinement orient first before changing their shape [20].

For flexible polymers, with $\varepsilon \geq 1$, the initial effect of confinement is to increase $R_{\|}$but decrease $R$. There is a clear dip in $\Delta R^{2}$, which can be explained by the following geometric argument. Consider an initially randomly oriented end-toend vector $\mathbf{R}$. Then weak confinement will predominantly reduce the magnitude of the component of this vector perpendicular to the tube axis but leave the parallel component unchanged. This obviously leads to a decrease in the magnitude of the end-to-end distance. We have checked this argument by measuring the projection of the mean-square endto-end distance onto the tube axis (data not shown), which indeed does not show an initial decrease but increases monotonically. A dip in the radius of gyration $R_{G}$ (not the end-toend distance) has previously been reported for very long $(\varepsilon \gg 1)$ self-avoiding polymers [20]. There it was argued to be a consequence of an intermediate regime, where the principal components of the radius of gyration tensor are reduced before confinement excluded-volume effects lead to an increase in $R_{G}$.

In the limit of strong confinement, where the Odijk deflection length is either much smaller than the total length (for stiff polymers) or much smaller than the persistence 


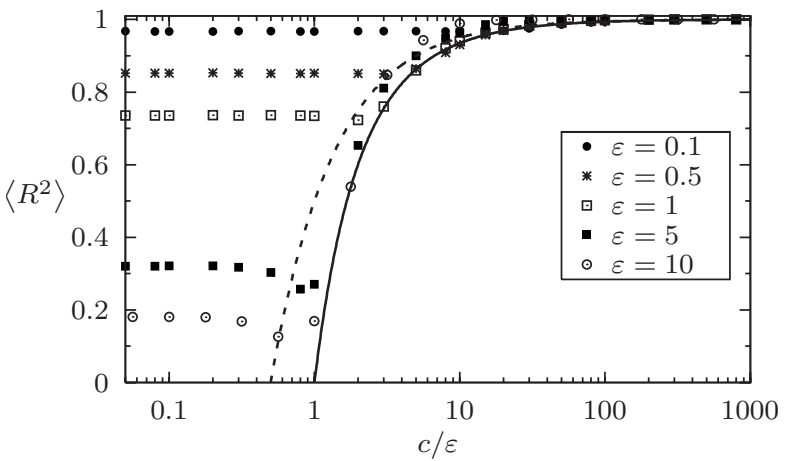

FIG. 4. Scaling plot for the mean-square end-to-end distance $\left\langle R^{2}\right\rangle$ versus $c / \varepsilon=\ell_{p} / L_{d}$ for various flexibilities $\varepsilon$. Universal scaling curve as estimated from the simulation (solid line) and obtained in the WBR limit (dashed line).

length (for flexible polymers), one expects $\left\langle R^{2}\right\rangle$ to become independent of the filament length. In order to show this explicitly we have replotted our MC data in Fig. 4 as a function of the number of collisions within a persistence length, $c / \varepsilon=\ell_{p} / L_{d}$. Indeed, all curves for different flexibilities merge into a master curve for strong confinement. In contrast to the master curve for weak confinement, which applies only for stiff chains, this master curve captures all chain flexibilities. The numerical result asymptotes the analytical formula obtained in the WBR limit, but only for $c / \varepsilon$ quite large. This shows that for intermediate confinement the orientation and the less constrained ends of the filament con- tribute significantly to the conformations; both of these effects are neglected in the WBR limit.

Advances in microfabrication and nanofabrication technologies have made it possible to confine biopolymers to topographical structures whose dimensions are comparable to or even smaller than their persistence length. This opens a range of novel possibilities to visualize and manipulate DNA and cytoskeletal filaments. Here, we have presented an extensive theoretical analysis of the shape and conformations of biopolymers resulting from strong confinement and identified and quantified a range of novel scaling regimes. We make specific predictions for experimentally accessible quantities like the density profile or the orientation and apparent length of a biopolymer in a channel. Our calculations may provide a road map for a clear identification of the possible scaling scenarios involved in the manufacture of nanofluidic and microfluidic devices. At the same time, our analysis is a first step toward a quantitative master curve connecting the apparent length and the actual length of DNA in nanochannels, which has important implications for experimental realizations aimed at a rapid screening of entire genomes in contrast to gel electrophoresis. Finally, they shed some light on the effect of cellular crowding on the conformation of cytoskeletal filaments.

We thank Tobias Munk for helpful discussions. We acknowledge financial support from Marie Curie Contract No. MERG-CT-2004-513598 and PRIN 2005 (G.L.) and the DFG through Grant No. SFB 486 (E.F.). Financial support of the German Excellence Initiative via the program Nanosystems Initiative Munich (MIN) is gratefully acknowledged.
[1] M. M. A. E. Claessens et al., Nat. Phys. 2, 186 (2006).

[2] B. Hinner, M. Tempel, E. Sackmann, K. Kroy, and E. Frey, Phys. Rev. Lett. 81, 2614 (1998).

[3] R. Austin, Nat. Mater. 2, 567 (2003).

[4] W. Reisner et al., Phys. Rev. Lett. 94, 196101 (2005).

[5] J. O. Tegenfeldt et al., Proc. Natl. Acad. Sci. U.S.A. 101, 10979 (2004).

[6] S. Köster, D. Steinhauser, and T. Pfohl, J. Phys.: Condens. Matter 17, S4091 (2005).

[7] A. Balducci et al., Macromolecules 39, 6273 (2006).

[8] C. Bouchiat et al., Biophys. J. 76, 409 (1999).

[9] L. Le Goff, O. Hallatschek, E. Frey, and F. Amblard, Phys. Rev. Lett. 89, 258101 (2002).

[10] T. Odijk, Macromolecules 16, 1340 (1983).

[11] P.-G. de Gennes, Scaling Concepts in Polymer Physics (Cornell University Press, Cornell, 1979).

[12] L. D. Landau and E. M. Lifschitz, Lehrbuch der theoretischen Physik, Band V, Statistische Physik Teil 1 (Akademie Verlag,
Berlin, 1979).

[13] N. Saitô, K. Takahashi, and Y. Yunoki, J. Phys. Soc. Jpn. 22, 219 (1967).

[14] H. Yamakawa and M. Fujii, J. Chem. Phys. 59, 6641 (1973).

[15] J. Wilhelm and E. Frey, Phys. Rev. Lett. 77, 2581 (1996).

[16] We validated our MC simulation against the results for the tangent-tangent correlation and the radial distribution functions of the end-to-end distance (amongst others) in the case of an unconfined chain. Perfect agreement was obtained within statistical error with the Gaussian results for flexible chains and the results in [15] for stiff chains.

[17] J. A. Aronovitz and D. R. Nelson, J. Phys. (Paris) 47, 1445 (1986).

[18] C. Haber, S. A. Ruiz, and D. Wirtz, Proc. Natl. Acad. Sci. U.S.A. 97, 10792 (2000).

[19] W. Kuhn, Kolloid-Z. 68, 2 (1934).

[20] J. H. van Vliet and G. ten Brinke, J. Chem. Phys. 93, 1436 (1990). 